\title{
Précision de l'estimation de l'équivalent en eau de la neige obtenue avec la sonde SNOWPOWER au Québec (Canada)
}

\author{
Precision of estimated Snow Water Equivalent (SWE) derived from the new sensor \\ SNOWPOWER in Québec (Canada)
}

\author{
Mohamed Niang', Monique Bernier ${ }^{2}$, Eric van Bochove ${ }^{3}$, Yves Durand ${ }^{1}$ \\ ${ }^{1}$ Météo-France/CEN, 1441 rue de la Piscine, Dom. Univ., 38406 St. Martin d'Hères cedex, France \\ Tél : + 33 (0) 4766337 60, Fax : e-mail : mohamed.niang@meteo.fr, \\ 2 INRS-ETE, 90 de la Couronne, Québec (Québec), G1K 9A9 \\ Tél : +1 418654 2585, Fax : +1 418654 2600, e-mail : Monique_Bernier@ete.inrs.ca \\ ${ }^{3}$ Agriculture et Agroalimentaire Canada, 2560, boul. Hochelaga, Sainte-Foy (Quebec), Canada, G1V 2J3, \\ Tél : +1 418 657-7985 poste 229 Fax : + 1418 648-2402 e-mail : vanbochovee@agr.gc.ca
}

This paper is about the accuracy gotten to determine the Snow Water Equivalent (SWE) and the snow moisture using a new in situ sensor, the "SNOWPOWER" probe. This sensor can estimate simultaneously the snow density and the snow moisture by measuring the dielectric constant of the snow at low and high frequencies along a 10 to 20-m long flat-band cable. The high frequencies signal is measured with a Time Domain Reflectometer (TDR), the low frequencies signal is measured with an impedance analyser. The proposed original methodology allows also to extract the snow height and then to use the sensor for SWE estimation. This paper gives the results of one experimental season, the winter 2003-2004, in a agricultural field located near Québec City in Canada $\left(48^{\circ} 28^{\prime} 12^{\prime \prime} N, 71^{\circ} 10^{\prime} 48^{\prime \prime} \mathrm{W}\right)$. The estimated derived from the new automatic sensor are compared with manual measurements of the snow properties. The results show a good estimation of the snow liquid water content $(0.40 \%$ volumetric) and the SWE $(14.07 \mathrm{~mm})$. However, results show the importance of the selection of the low frequency and of the algorithm for the snow temperature extrapolation for the accuracy of the estimation. Finally, we discuss the integration of the sensor's densities to the EQeau model for mapping the SWE using radar satellite data.

\section{INTRODUCTION}

Plusieurs efforts ont été investis durant cette dernière décennie sur la caractérisation physique du manteau neigeux aussi par des méthodes in situ que par la télédétection spatiale. La complexité de la neige réside dans le fait qu'elle est sans cesse en métamorphose et ses propriétés physiques et diélectriques sont en perpétuelle évolution, ce qui rend relativement difficile la mesure de ses propriétés. Généralement, l'Équivalent en Eau de la Neige (ÉEN) et la Teneur en Eau Liquide (TEL) sont les deux paramètres les plus importants en hydrologie des milieux nordiques et en météorologie, particulièrement pour la prévision des apports en eau, la gestion des grands complexes hydroélectriques, la prévision des risques naturelles (inondations, avalanches).

Ces deux grandeurs sont généralement mesurées indépendamment ou estimées à l'aide des modèles hydrologiques à l'échelle des bassins. La méthode manuelle d'échantillonnage de neige sur le terrain est très coûteuse notamment pour des zones éloignées difficiles d'accès et de grande étendue. De plus, pour une couverture neigeuse naturelle avec des grandes variabilités spatiales, cette méthode s'avère peu fiable pour fournir des valeurs représentatives notamment à cause de la faible résolution des données, leur faible volume et leur discontinuité [Martin, 1999]. De ce fait, leur confrontation avec des données intégrées de la télédétection spatiale ou avec des données issues de la modélisation numérique d'un manteau neigeux n'est pas souvent aisée. C'est dans la perspective d'assimilation des méthodes in situ à la modélisation numérique ainsi qu'aux données de télédétection spatiale qu'une nouvelle sonde [Brandelik \& Huebner, 1998], surnommée SNOWPOWER, a été développée dans le cadre du projet européen SNOWPOWER (NNE5/2000/251) [Stähli \& al., 2005].

De longueur variant de 10 à $20 \mathrm{~m}$ selon le besoin, cette sonde permet de déterminer simultanément la densité moyenne et la TEL sur une zone comparable à la résolution des capteurs radar souvent utilisés dans la mesure des propriétés de la neige comme ceux des satellites radar ERS-1,2, RADARSAT 1,2, ENVISAT. Dans cette étude, nous présentons les performances de cette nouvelle technique dans un contexte nordique de faible enneigement (Canada) durant l'hiver 2003-2004. Nous exposerons la méthode originale utilisée pour l'extraction de l'épaisseur de neige, un paramètre complémentaire de l'EEN. Par la suite, nous discuterons sur le potentiel de la sonde comme instrument opérationnel pour la cartographie de l'EEN à partir d'images radar et sur 
modèle d'estimation de l'EEN, le modèle EQeau [Bernier \& Fortin, 1998].

\section{LES DONNÉES ET LE PROTOCOLE EXPÉRIMENTAL}

Le site d'étude est le site expérimental d'Agriculture et Agroalimentaire Canada situé dans le bassin versant du Bras d'Henri, Québec, Canada (48²8'12” N, 71¹0’48” W). C'est un champ agricole avec des légères irrégularités de surface (figure 1). La sonde SNOWPOWER a été installée dès l'automne 2003-2004, et est graduellement recouverte de neige durant tout l'hiver. Ainsi la méthode est non destructive et la structure naturelle du manteau n'est pas modifiée.

Le protocole d'installation est bien décrit dans Niang \& al. [2005] et Stähli \& al. [2005]. De façon sommaire, la sonde a été installée diagonalement de façon à mesurer les propriétés verticales de la neige. La dimension horizontale du câble (c'est un câble de $15 \mathrm{~m}$ qui a alors été testé) permet d'intégrer l'information spatiale de la couverture nivale (figure 1).

La collecte des données de la sonde a été effectuée hebdomadairement depuis le 17 février 2004 jusqu'à la fin mars 2004. A chaque date, plusieurs mesures sont effectuées de façon continue. Parallèlement, des carottes de neige mesurant la hauteur et la densité moyenne de la neige sont effectuées à environ $1 \mathrm{~m}$ autour des câbles SNOWPOWER. Un profil vertical (fosse) donnant la température la densité et la TEL à chaque $10 \mathrm{~cm}$ selon l'épaisseur de la neige est également effectué à coté de l'installation. Sur le site une station météorologique permet d'enregistrer quotidiennement la température du sol, de l'air et la vitesse du vent.

Le mois de février 2004 est marqué par l'absence de pluie, la température de l'air est restée en dessous du point de congélation et le manteau neigeux est resté sec et dur. Cette période correspond à une période d'accumulation où l'épaisseur de neige avait atteint son maximum $(82 \mathrm{~cm})$. Les premières précipitations de pluie n'apparurent qu'en début mars suivies d'une hausse de température. Par la suite, l'initiation de la fonte printanière a commencée dès la deuxième quinzaine du mois de mars jusqu'à la fin du mois d'avril. A noter également qu'à cause des vitesses de vent élevées (car le site qui est situé en plein air), l'hiver 2003-2004 a été marqué par des densités de neige élevées $(>300 \mathrm{~kg} / \mathrm{m} 3)$.

\section{LA SONDE SNOWPOWER}

La conception technique et le principe de fonctionnement de la sonde SNOWPOWER ont déjà été présentés en détails [Niang \& al., 2003 et 2005 ; Brandelik \&Huebner, 1998 ; Huebner \& al., 1997]. D'une manière générale, le principe physique de la sonde est basé sur le fait que le contraste diélectrique de la glace entre les hautes et basses fréquences (dans le domaine des $\mathrm{kHz}$ ) est très significatif. De ce fait, il est possible de déterminer les contenus volumiques des trois constituantes de la neige (l'eau, l'air et la glace) par l'utilisation de deux fréquences. La constante diélectrique de la neige le long du câble est mesurée en haute fréquence par l'utilisation d'un TDR (la principale fréquence est de l'ordre de $87 \mathrm{MHz}$ ) et en basse par un analyseur d'impédance (fréquence variant de 1 à $300 \mathrm{kHz}$ ). Il est important de noter que la constante diélectrique de la neige entourant le câble n'est pas mesurée directement. Elle est déduite des fonctions de calibration déterminées par des mesures au laboratoire des matériaux dont on connaît la constante diélectrique à chaque fréquence. Il est également à noter que les propriétés diélectriques de la glace varient non seulement avec la température mais aussi avec la fréquence. D'où l'importance d'acquérir la température de la neige le long du câble et de savoir comment l'intégrer pour obtenir une donnée représentative. Enfin, étant donné que seulement deux fréquences sont requises pour déterminer la TEL et la densité, nous discuterons au paragraphe $\mathrm{V}$ de la nécessité d'utiliser le TDR et une fréquence basse au lieu de deux fréquences basses.

\section{DÉTERMINATION DE LA HAUTEUR DE NEIGE}

La sonde SNOWPOWER conçue pour la détermination simultanée de la TEL et la densité de la neige, peuvent aussi fournir la hauteur de la neige au fur et à mesure qu'elle s'accumule. Cela est rendu possible grâce à la configuration diagonale de l'installation (figure 2) et l'utilisation des données du TDR. En effet, le câble SNOWPOWER est une ligne de transmission. Le générateur de signal du TDR émet une impulsion électromagnétique de type échelon qui se propage via un câble coaxial le long des guides d'ondes constitués par le câble SNOWPOWER. Arrivé à l'extrémité finale des

\section{Direction des vents dominants}
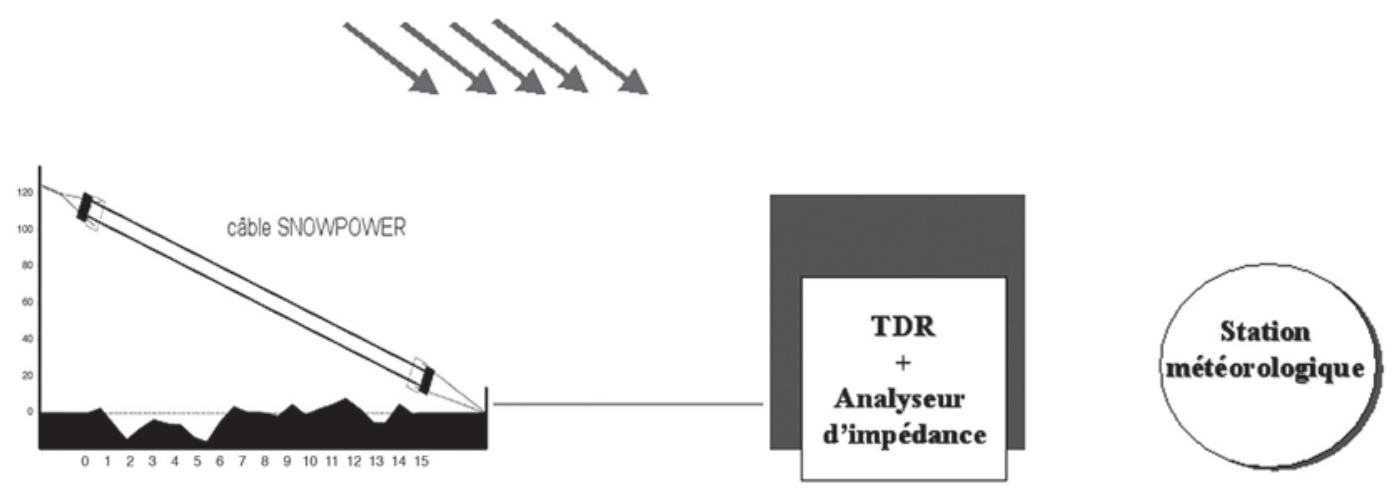

Figure 1 : Représentation schématique de l'installation des câbles de la sonde SNOWPOWER 


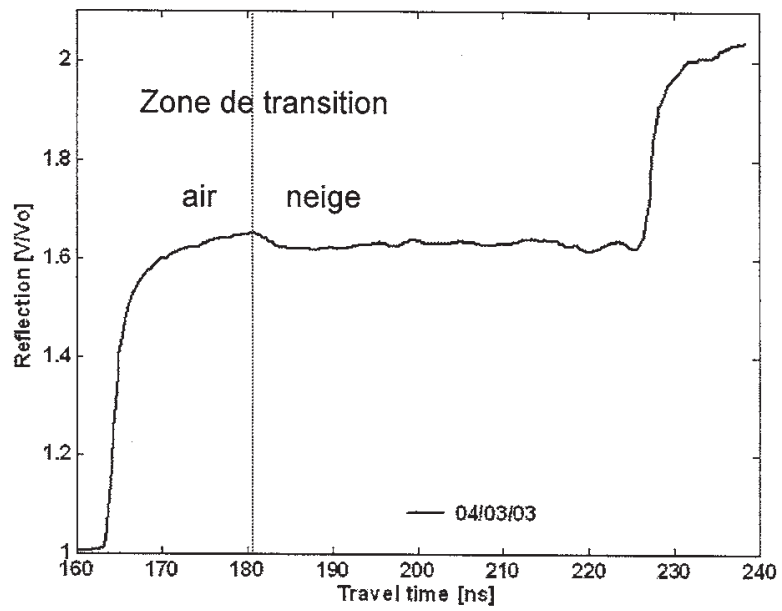

Figure 2 : Courbe du TDR du 03 mars 2004 sur laquelle est représentée le point de transition (barre verticale).

câbles, le front d'onde est réfléchi et repart vers l'oscilloscope qui enregistre les variations d'amplitude du signal en fonction du temps, en début du câble. Les signaux réfléchis auront des amplitudes variantes (en Volt) en fonction du type de discontinuités électriques rencontrées sur les câbles. Ce qui permet de détecter les variations diélectriques du manteau de neige. La courbe (ou trace) du TDR est généralement représentée par les variations du coefficient de réflexion $v$ (qui est le rapport des signaux réfléchis à ceux incidents) en fonction du temps $t$. La courbe du TDR exhibe souvent une ou plusieurs zones de transition dans le long du câble. Ces transitions sont dues aux pertes de puissance du signal. Parmi ces zones, la première est à l'entrée du câble dans la neige (à l'interface air-neige) à cause du contraste diélectrique entre ces deux milieux (figure 3 ).

La procédure de la détection des extremums locaux sur la courbe du TDR est conduite comme suit :

$$
f=\frac{d v}{d t}
$$

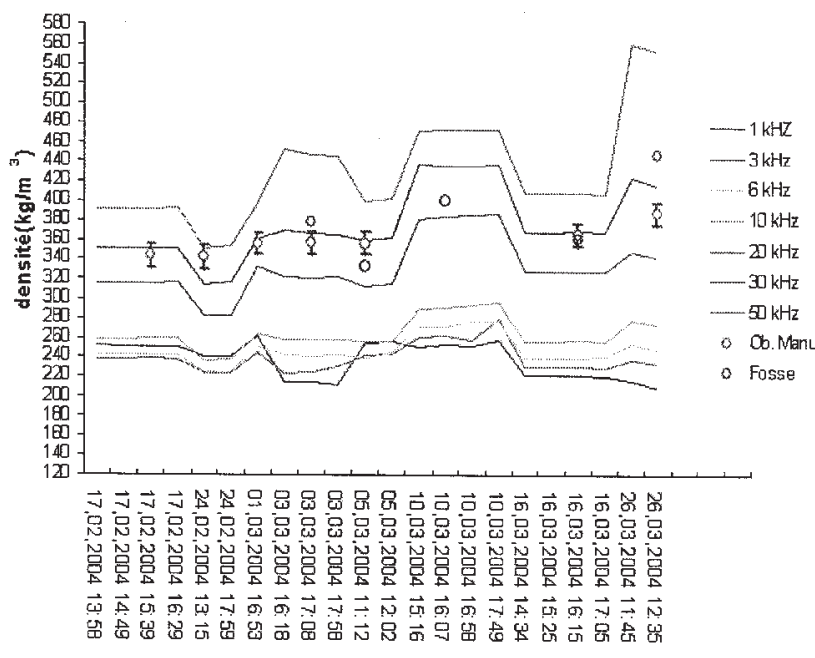

Figure 3 : Comparaison entre les densités simulées et celles mesurées manuellement pour différentes fréquences.
Il s'agit ensuite de pondérer cette fonction (1) par un poids qui pénalise la détection de la dernière transition au début de la courbe

$$
\operatorname{Max}\left[\left(y_{i-1}-y_{1}\right) \times(m-i)\right]
$$

avec $y_{i}=\operatorname{extremum~à~la~position~} i$ et $m=\max (i)$

Liées aux variations sur la courbe du TDR, cette fonction de pondération peut être l'une de ces équations: $(m-i)^{2}$, $\log (m-i)$ ou $\exp (m-i)$.

Le point de transition permet la détection de la longueur électromagnétique du câble enfouie dans la neige $L_{e}(x)$ avec laquelle on peut déduire l'épaisseur de neige $d(x)$ avec la configuration diagonale. Dans le cas d'une surface lisse.

$$
\sin \alpha=\frac{H}{L_{c}}
$$

et

$$
d(x)=\sin \alpha \cdot L_{e}(x)
$$

où $\alpha$ est l'angle formé par le câble et la normale. Cet angle est supposé constant à cause de la rigidité de l'installation.

$H$ étant la hauteur effective du support du câble et $L c$ la longueur du câble.

Dans le cas d'une surface avec des irrégularités de surface, l'épaisseur de neige est obtenue par une simple interpolation des épaisseurs de neige connues aux différentes positions du câble sans neige. Ces données ont été acquises après la fonte de la neige.

$$
d(x)=\operatorname{int} \operatorname{erpol}\left(L_{c}, H, L e(x)\right)
$$

C'est l'expression qui a été utilisée dans cet article.

\section{RÉSULTATS ET DISCUSSION}

\section{VI.1 Influence de la fréquence}

Comme cité dans le paragraphe III, nous allons étudier l'influence de la basse fréquence sur les valeurs estimées. La figure 3 montre une comparaison entre les densités simulées (avec une haute fréquence TDR et basse fréquence) et les données obtenues des observations manuelles. Les basses fréquences testées sont $1,6,10,20,30,50$ et $200 \mathrm{KHz}$. Nous constatons que de 1 à $10 \mathrm{KHz}$ les variations du signal basse fréquence sont faibles, ceci peut s'expliquer d'une part par le faible le rapport signal/bruit de l'analyseur d'impédance. D'autre part, les interférences causées par des appareils de navigation émettant sur des fréquences voisines -peuvent affecter le signal à ces fréquences. Sur la figure 3 , nous constatons que les observations manuelles (ob. Manu.) et les profiles verticaux (fosse) s'accordent bien aux densités simulées à la fréquence de $30 \mathrm{KHz}$. C'est cette fréquence qui va être utilisée dans cette étude.

Pour analyser la nécessité d'utiliser les données TDR, nous avons effectué une comparaison entre les valeurs estimées à partir de deux basses fréquences $(30+200 \mathrm{KHz})$ et celles estimées à partir d'une basse fréquence et d'une autre haute $(30 \mathrm{KHz}+\mathrm{TDR})$. Les résultats montrent (figure 4) que lorsque le couvert nival est sec (du 17 au 24 février) la diffé- 


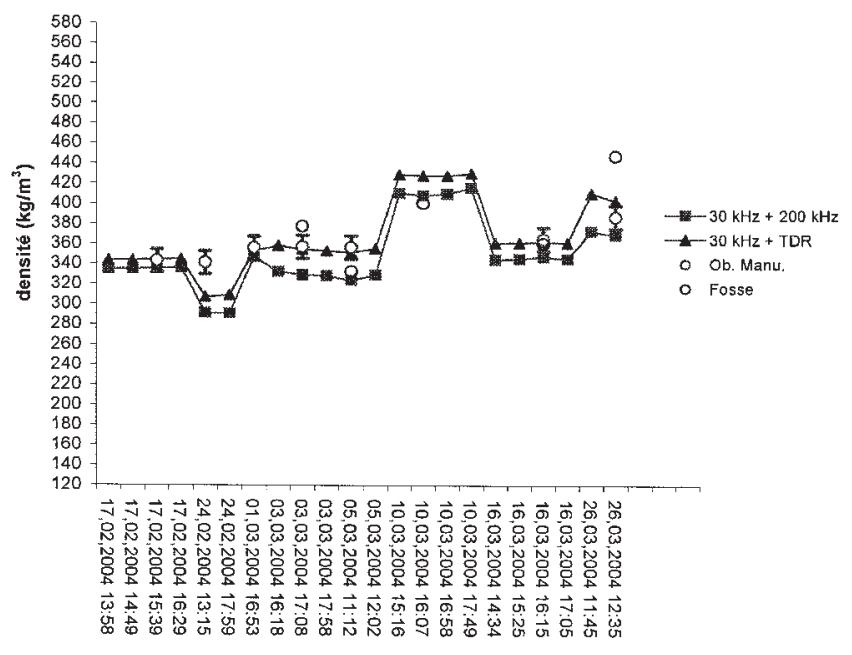

Figure 4 : Comparaison entre les densités simulées et celles mesurées manuellement différentes fréquences.

rence entre les deux méthodes est faible (moyenne de $10 \mathrm{~kg}$ $\mathrm{m}^{-3}$ ) tandis qu'elle peut atteindre $32 \mathrm{~kg} \mathrm{~m}^{-3}$ durant la période de pluie ( 3 mars) et de fonte ( 26 mars).

Pour la TEL, nous constatons que l'utilisation des fréquences basses n'est pas adéquate. Ces fréquences surestiment la TEL (figure 5). Cependant, la combinaison de fréquences $(30 \mathrm{KHz}+\mathrm{TDR})$ fournie une très bonne corrélation entre les valeurs estimées et les données manuelles. De plus, la date de la petite pluie (le 3 mars) ainsi que celle de la fonte intensive (le 26 mars) sont bien visibles sur le graphique. Une erreur absolue de $0.55 \%$ et une erreur relative de $0.40 \%$ ont été obtenues.

\section{VI.2 Influence de la température}

La densité de la neige est fortement influencée par la température du profil de neige. En effet, un changement de température produit un changement de la structure de la neige avec l'augmentation de sa TEL ce qui influence les propriétés diélectriques de la neige donc sa densité. A titre d'exemple, une variation de température de $\pm 2{ }^{\circ} \mathrm{C}$ peut faire varier la densité de $\pm 2,84 \mathrm{~kg} \mathrm{~m}^{-3}$ pour la neige sèche, et de $\pm 6,42 \mathrm{~kg} \mathrm{~m}^{-3}$ pour la neige humide. Cette dépendance entre la densité de la neige et de sa température affecte seulement les données basses fréquences. Les données hautes fréquences sont insensibles à la température. Nous avons aussi analysé la problématique d'intégration de la température d'un profil de neige dans le calcul de la densité et de la TEL. Il a été montré que la température moyenne n'était pas représentative des températures du manteau neigeux en conditions nordiques (couvert nival peu épais et températures de l'air très basses). Une méthode d'intégration de la température du manteau en fonction de l'épaisseur de neige a alors été développée [Niang \& al.,2005; Stähli \& al., 2005]..C'est ce modèle qui a été utilisé ici (Figures 5 et 6 ).

\section{V.3 Extraction de l'épaisseur de neige}

La figure 7 montre la comparaison entre les épaisseurs de neige estimées avec le modèle décrit au paragraphe 5 et celles obtenues par observations manuelles. D'une manière

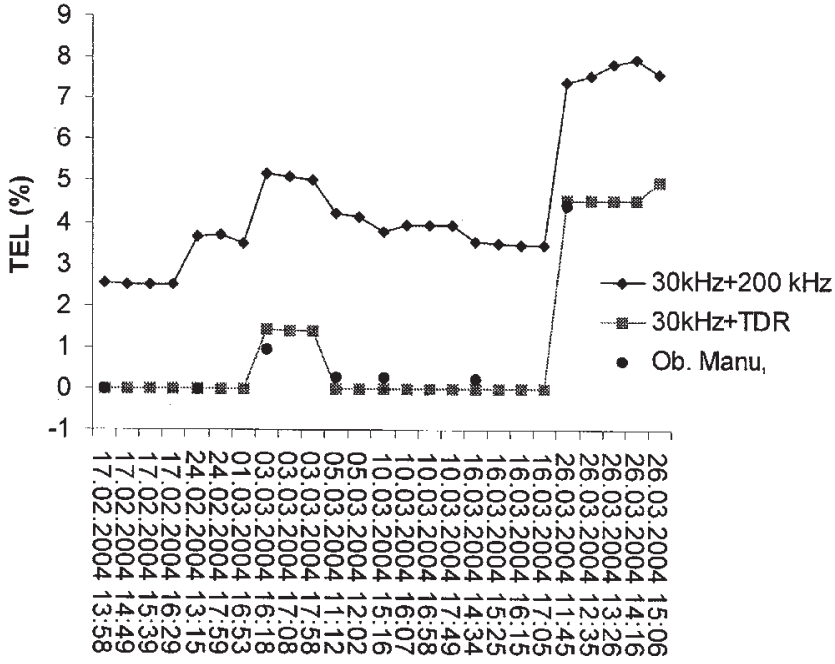

Figure 5 : Comparaison entre les TEL estimées et celles mesurées sur le terrain pour deux combinaisons de fréquences.

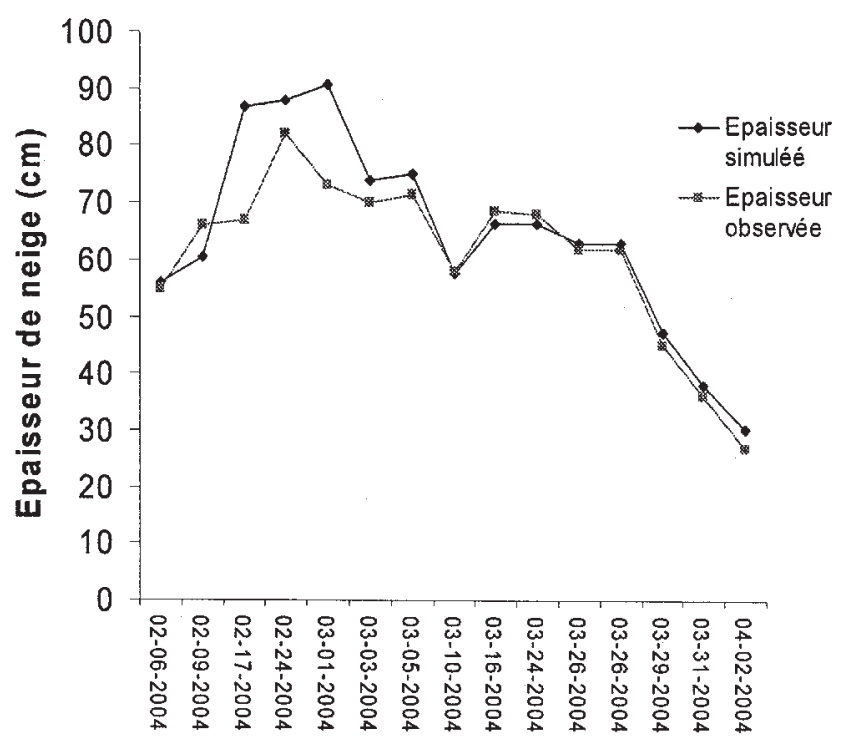

Figure 6 : Comparaison entre les épaisseurs de neige estimées et celles mesurées sur le terrain.

générale, la corrélation est très bonne entre les deux types de données. Néanmoins, on voit que la différence la plus importante entre les deux courbes est observée durant la période d'accumulation : le 17/02 (épaisseur $=67 \mathrm{~cm}$, différence $=19,95$ ), 24/02 (épaisseur $=82 \mathrm{~cm}$, différence $=6,04$ ) et le $01 / 03$ (épaisseur $=73, \mathrm{~cm}$ différence $=17.75 \mathrm{~cm}$ ). Cette différence de hauteur pourrait s'expliquer par le fait que le carottier n'ait pas pu atteindre le sol à cause d'une couche de glace située à environ $10 \mathrm{~cm}$ du sol. Elle pourrait aussi être dû à la précision de la détermination du point de transition. En effet, comme le montre les 3 courbes du TDR en condition de neige sèche (figure 7a), il est difficile de détecter la transition avec précision car le contraste diélectrique entre la neige sèche et air est faible. Par contre, en neige humide ce contraste augmente et rend la détection plus précise (figure $7 \mathrm{~b}$ ). 


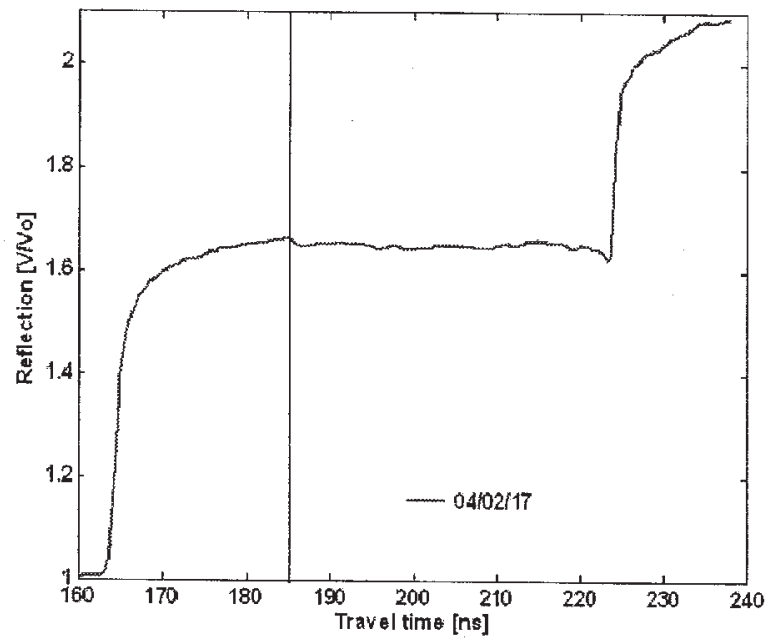

a) Courbe TDR du 17/02/2004 (neige sèche)

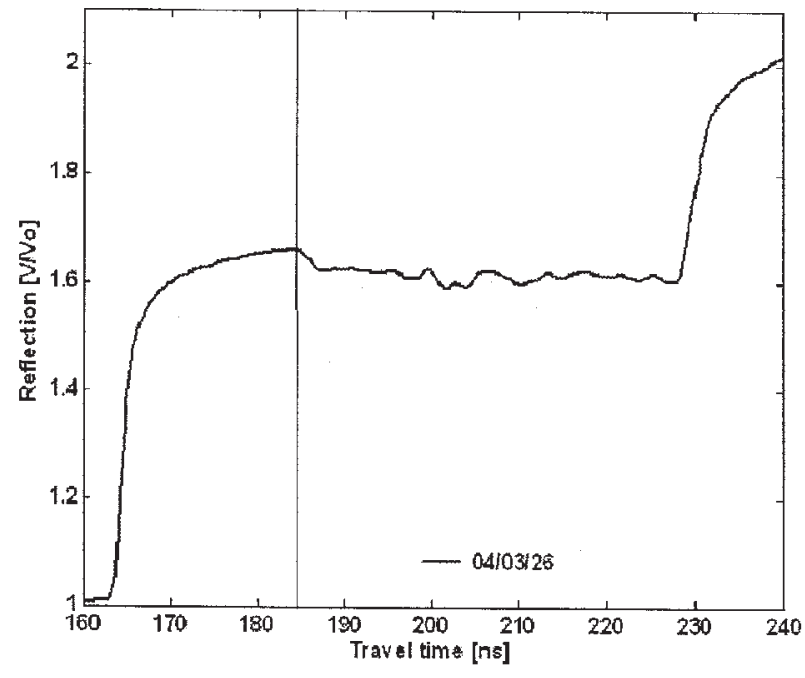

b) courbe TDR du 26/03/2004 (fonte)

Figure 7 : Comparaison entre les courbes du TDR a) d'une neige sèche et b) celle d'une neige humide.

\section{V.4 Equivalent en eau de la neige}

La figure 8 montre que la sonde SNOWPOWER fournit une bonne estimation de l'EEN. Mis à part les dates d'accumulation où la mesure manuelle peut être défaillante et où la détection du point de transition peut ne pas être très précise, les données estimées sont en accord avec celles manuelles. En général, la sonde SNOWPOWER surestime très légèrement l'EEN. L'erreur absolue est $14.07 \mathrm{~mm}$ et l'erreur relative est de 5.76 .

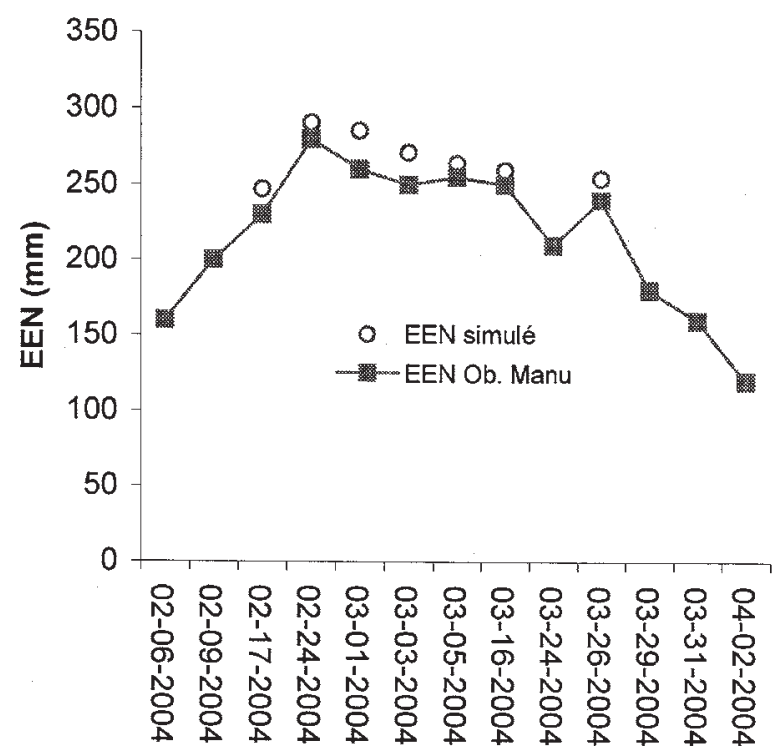

Figure 8 : Comparaison entre les EEN estimés et ceux simulés.

\section{V.5 Mise en relation avec les données radar}

Les capteurs radar dans le domaine des micro-ondes actifs ce sont avérés être les instruments les plus appropriés dans la surveillance et la caractérisation d'une couverture nivale des zones étendues et éloignées puisqu'ils acquièrent des ima- ges indépendamment des conditions météorologiques ou de l'éclairement solaire. Actuellement, les capteurs radar embarqués sur des satellites (ERS-1 et 2, Radarsat-1, ENVISAT) fonctionnent uniquement en bande $\mathrm{C}(5 \mathrm{GHz})$. Dans cette bande de fréquence, seul le modèle semi-empirique EQeau [Bernier \& Fortin, 1998] permet l'estimation de l'EEN dans certaines conditions environnementales. Le fondement de ce modèle est que la résistance thermique du couvert de neige influence les caractéristiques diélectriques du sol sous-jacent lorsqu'il est gelé et qu'elle peut être reliée au rapport de rétrodiffusion d'une image d'hiver et d'une image d'automne en supposant des conditions de rugosité identiques. L'EEN est déduit de la résistance thermique en tenant compte de la densité. Une analyse d'incertitude sur les données d'entrée et les paramètres du modèle a montré que la densité est le paramètre d'entrée le plus sensible du modèle EQeau [Stähli $\&$ al, 2005 ; Vincent \& al, 2004]. L'incertitude sur les valeurs de densité de la neige est aussi la principale source d'erreur sur l'estimation de l'équivalent en eau. Afin de réduire l'incertitude sur l'ÉEN, il faut, à la fois, réduire l'incertitude sur la mesure locale $\left(11.5 \mathrm{kgm}^{-3}\right)$ et sur l'interpolation régionale $\left(23 \mathrm{kgm}^{-3}\right)$. La réduction de l'incertitude locale pourrait être obtenue en utilisant des instruments automatiques plus précis que les mesures manuelles. La sonde SNOWPOWER serait un bon candidat car l'expérience de l'hiver 2003-2004 a permis d'obtenir une erreur absolue inférieure à $9 \mathrm{kgm}^{-3}$ pour les mesures de densité [Niang et al.,2005] . Quant à l'incertitude régionale, elle pourrait être réduite en utilisant des méthodes d'interpolation plus efficace comme le Kriging .

\section{CONCLUSIONS}

Cette étude a montré les performances de la sonde SNOWPOWER dans l'estimation de la TEL et dans l'EEN. Pour ce qui de la TEL, la combinaison d'une fréquence basse et le TDR est nécessaire pour obtenir une estimation précise. Concernent la densité, l'influence de la fréquence et de la température sont importantes sur les données basse fréquence. La méthode originale utilisée pour l'estimation 
de l'épaisseur de la neige a fourni de bons résultats en comparaison avec les données in situ. Finalement, la sonde SNOWPOWER est outil non destructif très performant pour l'estimation des deux paramètres les plus importants de la neige et pourrait également être utilisé dans le futur en combinaison avec des images de télédétection radar pour la cartographie de l'ÉEN.

\section{REMERCIEMENTS}

Ce travail a été effectué dans le volet canadien du projet international SNOWPOWER financé par le $5^{\text {ième }}$ programme cadre européen (NNE5/2000/251) et Recherche en Sciences et Génie Canada. Les auteurs tiennent à remercier tous leurs collègues et partenaires institutionnels du projet. Ils tiennent à remercier en particulier Yves Gauthier et Stéphane Savary de l'Institut National de la Recherche Scientifique - centre Eau, Terre et Environnement (INRS-ETE) pour la collecte des données terrain et Karem Chokmani, également de l'INRS-ETE, pour l'analyse d'incertitude des paramètres d'EQeau.

\section{RÉFÉRENCES}

Bernier, M., \& Fortin, J. 1998. The potential of times series of C band SAR data to monitor dry and shallow snow cover, IEEE Trans. Geoscience and Remote Sensing, 36, 226-246.

Brandelik A, \& Huebner C. 1997. Large-area, long-term monitoring of mineral barrier materials: Proceedings of the 1997 International Containment Technology Conference and
Exhibition, St. Petersburg, Florida, February 9-12; 10601066.

Hübner C, \& Brandelik A. 2000. Distinguished Problems in Soil and Snow Aquametry: Sensors Update, Chapter 13, Baltes, H., Goepel, W. and Hess, J. (Eds.), 7 : 317-340.

Martin, D. 1996. Analyse multicritère de capteurs satellitaires et aéroportés pour le suivi du couvert Nival. In Proceeding of the Eastern Snow Conference, Williamsburg (USA), May 1996 (New Hamshire : U.S. Army CRL), 67-81.

Niang, M., Bernier M., Stacheder M., Brandelik A., \& van Bochove E. 2005 . Influence of snow temperature and dielectric mixingmodel coefficient on density and liquid water content determination in a cold seasonal snow pack. Subsurface Sensing Technologies and Applications An International Journal, (soumis).

Niang, M., Bernier, M., Gauthier, Y., Fortin, G., Martel L., van Bochove, E., Stacheder, M. \& Brandelik, A. 2003. On the validation of snow densities derived from SNOWPOWER probes in a temperate snow cover in Eastern Canada : first results. 60e Eastern Snow Conference, 04-06 June, 175-187.

Stähli, M., Gutafasson, D., Stacheder, M., Bernier. M., Niang, M., Chokmani, K., Savary, S., van Bochove, E., Sommer, W. \& I. Völksch 2005. Test of New In-Situ Snow Sensor for Validation of Remote Sensing Images. EAReL Proceedings, Berne, 21-23 février 2005.

Vincent, P. Bernier. M., Carignan, M., Hardy, S., Gauthier Y., Chokmani, K., Niang. M, \& Khaldoune, J. 2004. EQeauPolar : Élargir l'application EQeau à l'utilisation des images ENVISAT et mesurer l'apport des données polarimétriques en vue de RADARSAT-2. Rapport Final présenté à l'ASC par Viasat Géo-Technologies (confidentiel). Rapport de recherche. INRS-ETE $\mathrm{N}^{\circ}$ R-783-f1. Novembre, 82 pages et 2 annexes. 


\section{Prochaines manifestations \\ de la Société Hydrotechnique de France}

\section{6}

- 31 mai $-1^{\text {er }}$ juin, Paris : Grandes crues historiques, $150^{\text {ème }}$ anniversaire des crues de 1856 (avec les DIREN Rhône Alpes, Centre et MidiPyrénées et l'AFPCN)

- 4 - 8 septembre 2006, Nice, Sessions spéciales du congrès Hydroinformatics 2006 de l'AIRH

- 17 - 18 octobre 2006, Nantes : JDHU 2006, 2èmes journées doctorales en hydrologie urbaine

- 19 octobre 2006, Paris : Journée au Sénat, $2^{\text {ème }}$ volet de l'anniversaire des crues de 1856, organisée par l'AFPCN, avec l'aide de la SHF.

- 5 Décembre 2006, Paris : participation à la session « Coastal risks » des Journées scientifiques et techniques du CETMEF

- 12 au 14 décembre 2006, Toulouse : $3^{\text {ème }}$ Congrès microfluidique

\section{7}

- Janvier 2007 : séminaire sur les pollutions marines

- Mars 2007 : 29 èmes Journées de 1'hydraulique : Le climat, ses variations séculaires et ses changements prévus: quel impact sur l'hydrologie et les évènements rares?

- Juin 2007 : Gestion dynamique des eaux souterraines (de l'anthropisation non contrôlée à la gestion concertée des aquifères) et Relations nappes/rivières

- Octobre 2007 : Gestion sociale et économique de l'eau

- fin 2007 : Perspectives de l'hydroélectricité (sous réserve)

\section{ఠ]}

Tous renseignements sur le site de la SHF

\section{www.shf.asso.fr}




\section{JDHU 2006}

2emes Journées Doctorales en Hydrologie Urbaine - JDHU 2006, organisées en partenariat avec la SHF - Société Hydrotechnique de France, I'ASTEE - Association Scientifique et Technique pour l'Eau et I'Environnement, I'AUGC - Association Universitaire de Génie Civil, I'IRSTV - institut de recherche sciences et techniques de la Ville et SAFEGE Ingénieurs conseils

Les $2^{\circ}$ Journées Doctorales en Hydrologie Urbaine (JDHU 2006) s'adressent à la fois

- aux étudiants en thèse, éventuellement en $2^{\circ}$ année de Master Recherche ou en post-doc, qui préparent leur diplôme en France ou à l'étranger en langue française,

- et à tous les enseignants-chercheurs, chercheurs et praticiens (publics, privés, gestionnaires, bureaux d'études, ministères, administrations, etc.) intéressés par les travaux de recherche en cours en Hydrologie Urbaine (au sens large) et par un lieu ouvert d'échange et de discussion.

Les JDHU 2006 ont pour objectif de permettre aux étudiants en thèse :

- de présenter leurs travaux (quel que soit le stade d'avancement dans leur thèse ou leur diplôme) à leurs collègues et à tous les chercheurs et praticiens intéressés, et de connaître ceux de leurs collègues ;

- de faire connaissance et de créer des liens entre eux, de manière à favoriser les collaborations entre personnes et entre institutions ;

- de s'entraîner à l'animation de séances scientifiques (présidence de session) et au compte-rendu (secrétariat de session);

- de publier des articles (après sélection et révision) dans la Houille Blanche et/ou TSM ;

- de prendre contact et de faire connaissance avec la communauté française des chercheurs et des praticiens en hydrologie urbaine.

C'est là une occasion importante de favoriser et de promouvoir les échanges d'informations et les collaborations entre thésards et laboratoires au niveau national et avec nos voisins francophones.

Envoyer les résumés avant le 5 juin 2006 à : jdhu@lcpc.fr

Consulter l'appel à communications complet sur le site www.shf.asso.fr 


\section{LA HOUILLE BLANCHE, Revue Internationale de l'Eau}

rappelle que tous les numéros de la collection sont disponibles

soit sous leur forme originale, soit en reproduction.

Les thèmes principaux de 1998 à 2005

(détail des articles sur notre site www.shf.asso.fr)

- Le risque de crue en région parisienne (2)

- Eau et développement durable

- Comportement dynamique des pompes et turbines hydrauliques

- Hydrodynamique des procédés industriels diphasiques

- Petite hydroélectricité : aspects environnementaux

- Petite hydroélectricite

- L'Ecole française de l'eau

- Agriculture et environnement

- Glaciologie, nivologie

- Crues de la normale à l'extrême

- La gestion des risques liés aux inondations rapides et lentes

- L'eau, la vie et l'environnement

- Interactions mécaniques entre fluides et structures

- Glaciologie et nivologie

- Protection des eaux souterraines

- Logiciels de mécanique des fluides

$2001 \quad$ - Logiciels de mécanique des fluides

- Protection des eaux souterraines

- Machines hydrauliques : instationnarités et effets associés

- L'eau en Afrique du Nord

- Autosurveillance en réseau d'assainissement

- Gestion des sédiments

- Glaciologie et nivologie

2002 - Imagerie satellitaire et radar au service de l'eau

- Conditions maritimes extrêmes

- Forêts et Eau

- Hydraulique des millénaires, les leçons de l'histoire pour construire le XXI ${ }^{\text {ème }}$ siècle

$\mathrm{N}^{\circ} 3$

- Variations climatiques et hydrologie

- Glaciologie et nivologie

- Forum Mondial de l'Eau

- Rencontres de Grenoble sur la petite hydroélectricité

- Etats qualitatif et quantitatif des Eaux souterraines : la directive cadre européenne

$\mathrm{N}^{\circ} 1$

$\mathrm{N}^{\circ} 2$

$\mathrm{N}^{\circ} 2$ et 3

- Eau et économie

- Microfluidique : micro-écoulements liquides et gazeux

$\mathrm{N}^{\circ} 3$

- Gestion du risque Eau en pays semi-aride (1)

$\mathrm{N}^{\circ} 6$

- Genèse des crues et des inondations (1)

$\mathrm{N}^{\circ} 6$

- Année internationale de l'Eau douce

- Aménagements

- Gestion du risque Eau en pays semi-aride (2)

$\mathrm{N}^{\circ} 6$

$\mathrm{N}^{\circ} 1$

$\mathrm{N} 1$

- Genèse des crues et des inondations (2)

$\mathrm{N}^{\circ} 1$

- Glaciologie et nivologie

- Modèles physiques

- Etiages et crues extrêmes en Europe : perspectives historiques

$\mathrm{N}^{\circ} 2$ et 3

$\mathrm{N}^{\circ} 3$ et 4

$\mathrm{N}^{\circ} 4,5$ et 6

$\mathrm{N}^{\circ} 6$

- Crues méditerranéennes

- Crues méditerranéennes

- L'eau et le monde vivant : dans la nature et dans le corps humain

$\mathrm{N}^{\circ} 1$ et 2

- Hydrologie urbaine

- Ecoulements diphasiques

$\mathrm{N}^{\circ} 5$ et 6

- Ressources en eau

- Microfluidique

Bon de commande à retourner à La Houille Blanche - SHF - 25 rue des Favorites, F 75015 PARIS

Tél. +33 (0)1 42509103 - Fax +33 (0)42 505983 - Mail LHB@shf.asso.fr

Nom/Name : Prénom/First Name :

Société/Company:

Adresse/Address

Code postal/Postal code:

Pays/Country:

Mail :

$\mathrm{N}^{\circ}$ et année(s)

Numéro(s) simple(s) au prix unitaire de TTC de

$\square$ France : 22 euros

$\square$ Etranger : 29 euros

Règle la somme de ...... $€ \square$ par chèque bancaire à l'ordre de la Houille Blanche

\section{Ville/City}

Tél/Phone :

Fonction/Function:

Fax :

$\square$ France : 32 euros

$\square$ Etranger : 40 euros

$\square$ par virement bancaire CAISSE EPARGNE lle de France Paris (agence de Vaugirard -

285 rue de Vaugirard - F75015 PARIS) -Compte 17515900000401884549349

(IBAN FR76 17515900000401884549349 - CEPAFRPP751) 